\title{
Microsimulación y Economía de la Salud
}

\author{
Nuria Badenes Plá \\ Universidad Complutense de Madrid
}

\begin{abstract}
Resumen
La microsimulación constituye una herramienta cada vez más utilizada en el ámbito económico en general, y comienza a utilizarse también en el de la Economía de la Salud. La microsimulación se puede llevar a cabo de forma independiente mediante la programación realizada por un investigador para la resolución de un problema particular, si bien cada vez más es más frecuente encontrar que los esfuerzos se aúnen y se construyan modelos por parte de equipos y se ofrezcan también para el uso general. La ventaja de la construcción de grandes modelos reside en que estos pueden ser mejorados, depurados e incluso integrados con otros modelos. La proliferación de modelos de microsimulación es por tanto un ejemplo de las ventajas de la división y especialización del trabajo, de manera que hoy en día no es necesario saber construir la herramienta para poder utilizarla, y los esfuerzos de los investigadores se pueden centrar en lo que cada uno hace mejor: programar, diseñar mejoras en los modelos de microsimulación, o aplicar los modelos para la toma de decisiones. Este trabajo revisa los modelos de microsimulación más difundidos que se pueden hallar en el ámbito de la Economía de la Salud explicando sus utilidades
\end{abstract}

Palabras clave: Economía de la salud, modelos de microsimulación I10, C81. Clasificación JEL: I10, C81.

\begin{abstract}
Microsimulation has become a usual tool in Economics in general, and it starts to be more common in Health Economics. It can be part of the programming carried out by a researcher in order to solve a specific problem although it is increasingly common to come across coordinated efforts where models are constructed by teams and offered to general use. The advantage of constructing large models is that these can be improved, refined or incorporated into other models. The proliferation of microsimulation models thus exemplifies the advantages of division and specialisation of work, in such a way that today is not necessary to know how to construct the tool in order to use it. Researchers' efforts can then be focussed on what each one does best: programming, designing improvements for microsimulation models or applying models for decision making. This work reviews the more spread models in Health Economics explaining their utilities.
\end{abstract}

Key words: Health Economics, microsimulation models

JEL Classification: Health Economics, microsimulation models, I10, C81.

\section{Introducción}

La microsimulación se ha revelado como una herramienta poderosa en muchas áreas de la Economía, de las cuales, la Economía de la Salud no es una excepción. La microsimulación se puede llevar a cabo mediante la programación realizada por un investigador para la resolución de un problema particular, si bien cada vez más es 
más frecuente encontrar que los esfuerzos se aúnen y se construyan modelos por parte de equipos. La ventaja de la construcción de grandes modelos reside en que estos pueden ser mejorados, depurados e incluso integrados con otros modelos. La proliferación de modelos de microsimulación es por tanto un ejemplo de las ventajas de la división y especialización del trabajo, de manera que hoy en día no es necesario saber construir la herramienta para poder utilizarla, y los esfuerzos de los investigadores se pueden centrar en lo que cada uno hace mejor: programar, diseñar mejoras en los modelos de microsimulación, o aplicar los modelos para la toma de decisiones.

En este trabajo se lleva a cabo una revisión de los modelos de microsimulación que se pueden utilizar en el ámbito de la Economía de la Salud en diferentes países. El capítulo se organiza como sigue: tras esta introducción, el segundo epígrafe establece los límites de lo que queda comprendido dentro del concepto de microsimulación en el ámbito de la Economía de la Salud. El tercer epígrafe repasa por países los modelos desarrollados por equipos. En particular, nos referimos a modelos hallados en Australia, Canadá, Estados Unidos, Europa en conjunto, Francia, Holanda, Noruega, Reino Unido y de uso general. En el cuarto epígrafe se muestran algunos ejemplos de trabajos que utilizan modelos de microsimulación construidos «a la carta» para la resolución de un problema particular o bien modelos que no se encuentran a disposición del público en general. En el quinto epígrafe se presentan las reflexiones finales.

\section{2. ¿Qué entendemos por microsimulación en Economía de la Salud?}

Los modelos de microsimulación son modelos informáticos que operan desde el nivel del individuo. Estos modelos simulan a partir del comportamiento individual de colectivos representativos lo que ocurre en poblaciones más extensas con el fin de obtener conclusiones aplicables a niveles de agregación mayores. A partir del comportamiento al nivel del individuo es posible incorporar respuestas diferentes, y esto es lo que hace distintos estos modelos de los agregados, en los que las variables explicativas representan propiedades de la colectividad.

Existen áreas de Economía Pública en las que la microsimulación ha sido ampliamente utilizada como instrumento en la toma de decisiones, por ejemplo en el análisis distributivo de políticas de gravámenes y transferencias. En otras áreas, como es la Economía de la Salud, la microsimulación comienza solamente a hacer progresos, si bien la aplicabilidad y el potencial de esta herramienta son fabulosos.

El área de Economía de la Salud es muy extensa y difícil de delimitar, y los esfuerzos en la creación de modelos son todavía modestos, por ello aclaramos que cuando nos referimos a microsimulación en este ámbito pensamos en modelos muy variados, en particular: modelos de financiación de sanidad y del gasto farmacéutico, modelos de gestión sanitaria, de coste efectividad, modelos que estudian la demanda sanitaria e impacto económico del comportamiento relacionado con la salud y el envejecimiento de la población, modelos que analizan los efectos de factores de riesgo y los tratamientos sobre enfermedades determinadas y su repercusión en mortalidad 
y gastos sanitarios. En muchas ocasiones no encontraremos modelos específicamente sanitarios sino modelos tax-benefit en los que se incluyen variables relacionadas con gasto o prestaciones sanitarias, o modelos de pensiones en los que el estado de salud es una variable más a considerar.

Visto el amplio ámbito de aplicación de la microsimulación en Economía de la Salud, no es de extrañar que los usuarios actuales y potenciales de esta relativamente nueva forma de trabajar sean muchos y de diversa índole: el sector público, el sector privado, individuos particulares, empresas, instituciones sanitarias, organizaciones o el ámbito académico. Cada vez más la toma de decisiones ha de sostenerse sobre las bases de la cuantificación de hipotéticos escenarios y esto es algo que interesa a todo el mundo.

\section{Modelos de microsimulación en Economía de la Salud: exposición por países}

Como ya se mencionó, cada vez es más común encontrar grupos de trabajo que aúnan esfuerzos en la construcción de modelos de microsimulación. Los modelos tax-benefit han sido pioneros en el desarrollo de la microsimulación. Aunque estos modelos pueden analizar simplemente los efectos del día después de una política determinada, también pueden ser mejorados de forma que se incluya la respuesta de los afectados por la política. En un principio, los modelos tax-benefit analizaron exclusivamente cuestiones distributivas relacionadas con impuestos y transferencias, si bien cada vez es más posible hallar modelos que incorporan comportamientos de los individuos ante determinada política relativa a la salud, o simplemente se crean modelos específicos para el análisis de políticas sanitarias.

De evolución más reciente son los modelos que analizan el coste en pensiones o sanitario debido al envejecimiento de la población, ya que implican un paso más al incorporar un componente dinámico. La construcción de modelos dinámicos no es tarea fácil y requiere un esfuerzo tanto en la propia construcción de los modelos como en la estimación de las probabilidades de cada estado futuro considerado. Esta puede ser una explicación a la proliferación de equipos de trabajo multidisciplinares y compuestos por investigadores de distintos países.

A continuación se detallan por países e instituciones los modelos de microsimulación de mayor relevancia relativos a Economía de la Salud. En muchos casos no se trata de modelos específicamente sanitarios, sino construidos para otro fin pero en los que hay cabida a la consideración de cuestiones relacionadas con gasto sanitario, seguros sanitarios o pensiones. No trataremos de dar una explicación exhaustiva de cómo funcionan los modelos o de cuáles son los supuestos que incorporan ${ }^{1}$, sino de dar una visión general de cuáles son las herramientas con las que se puede contar hoy en el ámbito de la Economía de la Salud.

Para ello se recomienda consultar Zaidi y Rake (2002). Para una revisión de modelos de microsimulación puede consultarse además Spielauer (2002a y b) 


\subsection{Australia}

El NATSEM (National Centre for Social and Economic Modelling) de la Universidad de Canberra ha comenzado a aplicar técnicas de microsimulación a temas relacionados con política sanitaria. Como se expone en Brown y Harding (2002) y Brown et al. (2004), desde 1997 se está desarrollando el modelo de microsimulación PBS (Pharmaceutical Benefits Scheme). Este modelo se basa en un modelo previo desarrollado también por el NATSEM, el STINMOD, un modelo estático para el análisis de políticas de gravámenes y transferencias. A éste se le añadieron los datos del National Health Survey (NHS) relativos al uso de fármacos recetados según características socioeconómicas. Así, teniendo en cuenta la edad, el género y el grado de subsidio de los pacientes a los que se prescriben los medicamentos, el modelo es capaz de calcular:

1. Gasto simulado en PBS según hogares con diferentes características

2. Gasto del Gobierno bajo el esquema de subsidio farmacéutico (PBS)

3. Resto del coste out-of-pocket (contribución por copago de los pacientes) para los dos grupos de consumidores en Australia: generales y los que disfrutan de una concesión.

También en el ámbito de NATSEM se encuentra el modelo de microsimulación dinámico, DYNAMOD-2, diseñado para proyectar 50 años hacia delante las características de la población australiana. (Véase King et al.(1999)). El modelo opera sobre un $1 \%$ de la población (150.000 observaciones) y genera las historias de la población teniendo en cuenta eventos demográficos (fertilidad, mortalidad, formación y disolución de parejas, así como fenómenos migratorios). También considera nivel educativo, participación laboral y renta.

Al margen del ámbito oficial y académico, la compañía Laerdal ubicada en Oakleigh ofrece con fines educativos varios modelos de microsimulación, entre los que se encuentran MicroSim Inhospital y MicroSim Prehospital. El primero de estos modelos de microsimulación sirve para optimizar los recursos económicos y formativos en un hospital, mientras que el segundo está especialmente indicado para la situación previa a la hospitalización (médicos de emergencia, paramédicos, ambulancias)

\subsection{Canadá}

Canadá ha desarrollado varios modelos de microsimulación relacionados con la Economía de la Salud. En el seno del Ministerio de Estadística, encontramos el POHEM (Population Health Model) que es estrictamente un modelo de salud. Además cuentan con LifePaths que a pesar de ser un modelo más general, puede utilizarse para propósitos de análisis de políticas de Salud.

POHEM es un modelo de microsimulación longitudinal de salud y enfermedad. Mediante el uso de ecuaciones de submodelos que ha desarrollado en Ministerio de 
Estadística canadiense, el modelo simula poblaciones representativas y permite la comparación racional de políticas sanitarias alternativas en una trama que captura las interacciones causadas por la enfermedad.

Lifepaths es un modelo longitudinal dinámico de individuos y familias. A través de ecuaciones de comportamiento que tiene incorporadas, así como una variedad de bases de datos de historias a nivel micro, el modelo crea muestras representativas estadísticamente que consisten en períodos de vida completos de los individuos. Las ecuaciones de comportamiento del modelo generan en resolución subanual, los eventos discretos que configuran la historia completa del individuo. Además de estas capacidades longitudinales, Lifepaths es capaz de analizar resultados afinados y representativos teniendo en cuenta un conjunto de solapamientos en cohortes desde 1971 en adelante. LifePaths se utiliza para analizar y desarrollar y calcular el coste de los programas que tienen esencialmente un componente longitudinal, en particular, aquellos que requieren evaluación a nivel del individuo o la familia. También se puede utilizar para analizar materias sociales de naturaleza longitudinal como justicia intergeneracional o el momento más adecuado para la asignación de ciertos recursos a lo largo de la vida.

Además de los modelos que acabamos de describir, el Ministerio de Sanidad canadiense cuenta con otros modelos HTSIM (Health and tax microsimulation model), y modelos de oferta y demanda PHARMSIM y HHR (Health Human Resources).

HTSIM permite simular cambios en el tratamiento fiscal de los gastos relacionados con la salud y determinar los efectos distributivos y los costes generados por dichos cambios. La ventaja de este modelo frente a los ya existentes reside en que proporciona una explicación mayor de los gastos sanitarios, tanto los que quedan cubiertos como los que no reconoce el sistema fiscal.

PHARMSIM es un modelo que se encuentra actualmente en construcción. El principal objetivo que se pretende con él es analizar los patrones de utilización de medicamentos y el impacto distributivo de los planes de seguro médico. Puede utilizarse en la dirección: http://www.cdl.edu/pharmsim/

Los modelos HHR de oferta y demanda también están actualmente en fase de construcción. El objetivo que se pretende con ellos es capacitar al Ministerio de Sanidad para proyectar una serie de ecuaciones complejas, incluyendo participación laboral, edad y sexo del personal laboral sanitario. Se pretende la construcción de un modelo no mecánico, sino que incorpore comportamiento de los agentes. El modelo de demanda HHR se prevé sea construido de acuerdo con la utilización de médicos, así como las demandas de los pacientes de otros profesionales sanitarios.

Canadá utilizó CORSIM — un modelo dinámico previamente desarrollado por Estados Unidos - y lo adaptó a los datos canadienses creando DYNACAN. Se trata de un modelo de microsimulación, estocástico, longitudinal y dinámico ${ }^{2}$. Mediante

2 Es un modelo estocástico basado en simulaciones Monte Carlo de generación de números aleatorios para la generación de los eventos, es longitudinal porque proyecta la vida de los individuos/familias (y sus pensiones) a lo largo de toda la vida, y dinámico porque considera que las características de las familias pueden evolucionar en el tiempo. Véase Morrison y Dussault (2000) 
el uso de DYNACAN, se pueden llevar a cabo simulaciones acerca de distintos aspectos demográficos, rentas y otras características, que permitan analizar la evolución en los contribuyentes y beneficiarios del sistema de pensiones. Así, es posible estudiar la sostenibilidad del sistema existente, y las consecuencias de actuaciones alternativas por parte de la Oficina de Desarrollo de Recursos Humanos de Canadá (HRDC), principal usuaria del modelo.

\subsection{Estados Unidos}

Jonathan Gruber, afiliado al MIT y al NBER ha desarrollado en el pasado reciente un modelo de microsimulación cuya primera versión se detalla en Gruber (2000). El modelo toma una muestra ${ }^{3}$ representativa de población norteamericana, que contiene información de la oferta aseguradora que ofrece el empleador, cobertura sanitaria, renta, demográficos y estado de salud declarado por los encuestados. Este modelo permite calcular los efectos de distintas alternativas de política de seguros sanitarios en la distribución del gasto sanitario y los costes sanitarios en el sector público y privado. El modelo asume determinado comportamiento de las empresas, las familias y los individuos ante cambios en los precios absolutos y relativos de los seguros sanitarios.

El Urban Institute (Washington) comenzó en los años setenta a desarrollar una herramienta de microsimulación dinámica, que se revisó durante la década de los ochenta y sigue mejorándose en la actualidad. El objetivo de esta institución era determinar cómo las políticas de jubilación vigentes y propuestas, las tendencias demográficas y las prácticas del sector privado influían en la seguridad y la toma de decisiones de los americanos de edad más avanzada. Con ello se proporcionaba una herramienta a los políticos para enfrentarse ante potenciales crisis. Esta herramienta se conoce como DYNASIM3, que constituye un modelo de microsimulación dinámico que analiza las consecuencias distributivas de jubilaciones y envejecimiento de la población en el largo plazo. Véase Favreault y Smith (2004). El modelo trabaja con una muestra ${ }^{4}$ representativa, a partir de la cual se simulan eventos demográficos como crecimiento poblacional, formación de familias, educación o salud. Aunque no se trata de un modelo estrictamente sanitario, aborda uno de los principales problemas de interés en Economía de la Salud como es el envejecimiento poblacional.

El Urban Institute cuenta también con el modelo TRIM3 (Transfer Income Model) concebido a partir de una versión previa de un modelo de microsimulación (RIM, Reforms and Income Manteinance) desarrollada en 1969. Este modelo estático pretende capacitar al investigador para el trabajo con microdatos en la simulación de una

3 La muestra procede del CPS (Current Population Survey) correspondiente al año 1997.

4 Los datos se refieren a familias e individuos tomados del Survey of Income and Program Participation panels en los años 1990 y 1993, que se va haciendo envejecer año a año. 
gran variedad de programas de rentas y gravámenes, pero incluye específicamente programas de salud que afectan a la población norteamericana. Esta es la razón por la que el Departamento de Salud y Servicios Humanos financió la iniciativa. En particular, TRIM3 permite simular alternativas de política referidas a los programas:

- Medicaid

- SCHIP (State Health Insurance Programs)

- Medicare

- ESI (Employer Sponsored-Health Insurance)

La Universidad de Cornell comenzó en 1987 el desarrollo de su modelo CORSIM, cuya versión más reciente, CORSIM 4.1., data de 2002. CORSIM ha servido de referencia para la construcción de otros modelos dinámicos como POLISIM de la administración de la Seguridad Social estadounidense, SVERIGE en Suecia y DYNACAN en Canadá. Se trata de un modelo dinámico relativo a la población estadounidense, que tiene en cuenta el pasado reciente para simular qué ocurrirá en el futuro, modeliza políticas gubernamentales y en especial las relativas a programas de Seguridad Social. Los datos que incorpora de individuos y familias se refieren a características demográficas básicas como nacimiento, muerte, matrimonio, divorcio, emigración e inmigración. También incluye niveles educativos, información económica, de renta y laboral, acumulación de activos y deuda y contribuciones a planes de pensiones. Este modelo ha sido utilizado ampliamente por la Administración de la Seguridad Social norteamericana.

\subsection{Conjunto de Europa}

Dentro del conjunto de modelos tax-benefit, EUROMOD se presenta como uno de los proyectos más ambiciosos en cuanto a cobertura, ya que incluye 15 países de la Unión Europea (Alemania, Austria, Bélgica, Dinamarca, España, Finlandia, Francia, Grecia, Holanda, Irlanda, Italia, Luxemburgo, Portugal y Reino Unido) que se coordinan mediante una Unidad de Microsimulación. Se trata de un modelo de microsimulación estático que permite estimar el impacto distributivo de las políticas de gravámenes y transferencias tanto en el nivel particular de cada país como en el nivel global europeo. No se concibe como un modelo de Economía de la Salud, si bien al evaluar políticas sociales consolidadas en la Unión Europea incluye algunos conceptos relacionados con la salud. Para todos los países mencionados es posible simular políticas relativas a contribuciones sociales y asistencia social, pero solamente existe la posibilidad de simular políticas relativas a discapacidad o pensiones para algunos países. (Shuterland, 2001).

Además de esta iniciativa aglutinadora de países, pueden encontrarse modelos de microsimulación con aplicación en el ámbito de la Economía de la Salud para algunos países europeos de forma individualizada. 


\subsection{Francia}

Aunque no se trata de un modelo sanitario, Francia también ha desarrollado, al igual que otros países europeos un modelo dinámico que le permita simular la sostenibilidad de las pensiones, DESTINIE. El problema es que los esquemas públicos y privados de pensiones funcionan de forma diferente, y en el modelo se asume que todo el mundo contrata un plan de pensiones de forma privada. DESTINIE se comenzó a desarrollar a finales de los noventa, pero se proyecta la introducción del Sector Público en el futuro.

\subsection{Holanda}

Jan Nelissen desarrolló un modelo de microsimulación para Holanda, NEDYMAS, (véase Nelissen (1993 y 1996)) cuyo objetivo es comparar el grado de redistribución vertical de los esquemas de Seguridad Social sobre la renta vital de cuatro cohortes de 10 años nacidos entre 1930 y 1960. Para ello se precisaba de una muestra de sección cruzada desde el momento en que la primera cohorte se incorporaba al mercado laboral. Como esos datos no estaban disponibles, se generaron de forma hipotética con las mismas características que las del censo. Este modelo es entonces parecido a los modelos de cohortes, aunque no asume un estado estacionario, sino que simplemente intenta modelizar las condiciones en las que los individuos viven (y vivirán). Comenzando así con datos de sección cruzada y simulando el nacimiento de futuras generaciones el modelo permite analizar el impacto redistributivo a lo largo de la vida de los modelos tax-benefit para un grupo de cohortes. Este modelo holandés no es específicamente una herramienta de Economía de la Salud, pero sí un instrumento útil para evaluar el sostenimiento del sistema de pensiones ligado al envejecimiento.

\subsection{Noruega}

El departamento de Estadística de Noruega cuenta con varios modelos de microsimulación energéticos, impositivos, macroeconómicos, regionales y de población. Dentro del conjunto de los modelos de población se encuentra MOSART, de microsimulación dinámico que permite proyecciones en el largo plazo y analiza cambios poblacionales, en fuerza de trabajo, ayudas públicas a la educación y beneficios de la Seguridad Social. El modelo fue concebido por Fredriksen (1998) en el seno del Ministerio de Estadística noruego, con el fin de ayudar al estudio de opciones ante el reto de financiación del gasto público en el futuro. Los datos de los que se nutre el modelo suponen el $12 \%$ de la población noruega y es a partir de esos mismos datos de donde se estiman probabilidades de transición a distintos estados como discapacidad, rehabilitación, participación laboral. 


\subsection{Reino Unido}

El Departamento de Política social de la London School of Economics junto con el Instituto Gerontológico del King's College londinense y El Instituto de Ciencias de la Universidad de Southampton constituyeron SAGE (Social Policy in an Ageing Society) en Noviembre de 1999. El objetivo de este grupo es investigar el futuro de la política social en una sociedad que está envejeciendo. Una de las claves para lograr este objetivo reside en la construcción y uso de un modelo de microsimulación dinámico que facilite la evaluación del impacto de diferentes opciones de política. SAGE no está dedicada al estudio de la Economía de la Salud, pero entre las políticas analizadas que incluye en sus modelos se integra también la salud como variable a considerar Para una aplicación del modelo, véase Evandrou et al. (2001).

SAGE ha desarrollado una familia de modelos de microsimulación estática para evaluar políticas que impactan en rentas y pensiones en la última parte del ciclo vital, como RITA (Retirement Income Trajectory Analysis model). Este tipo de simulación se conoce como «hipotética» ya que permite conocer el gasto en pensiones bajo el sistema existente o bajo un rango de escenarios alternativos hipotéticos.

El grupo investigador de SAGE también ha construido un modelo de microsimulación dinámico SAGEMOD que permite proyectar una muestra de la población británica hasta 2020 simulando los principales eventos que acontecen en la vida de los individuos. Mediante los censos, datos de panel y datos de sección cruzada, los eventos simulados incluyen: nacimiento, muerte, estudios, matrimonio, divorcio, participación en el mercado laboral, obtención de rentas, estado de salud, jubilación, discapacidad y cuidado informal. Las probabilidades de que estos eventos ocurran se estiman a partir del estudio de probabilidades para cada uno de ellos de forma separada.

La Unidad de Microsimulación de la Universidad de Cambridge lleva a cabo la construcción de varios modelos de microsimulación con fines comparativos entre varios países. Uno de ellos es EUROMOD, expuesto anteriormente, y otro es POLIMOD diseñado para determinar los efectos en la distribución de la renta de los cambios en las políticas de gravamen sobre la renta y las políticas de Seguridad Social.

Un modelo más, aplicable al Reino Unido es PENSIM, concebido para proyectar las rentas de los pensionistas una vez jubilados. La primera versión del modelo dinámico se llevó a cabo por Hancock el al. (1992), posteriormente Curry (1996) desarrolló el modelo que actualmente utiliza el Ministerio de la Seguridad Social. Se piensa en ampliar para introducir en una nueva versión, PENSIM2, dimulación de discapacidad, entre otras potencialidades.

Un modelo específico del estudio de enfermedades coronarias en el ámbito del Reino Unido es el llamado Modelo de políticas de análisis de CHD. Comprende dos partes separadas. Una primera se desarrolló por la Escuela de Higiene y Medicina Tropical londinense para simular el efecto sobre costes y beneficios de diferentes estrategias preventivas. El componente de tratamiento del modelo se llevó a cabo por las Univer- 
sidades de Southampton y Birmingham, y permite evaluar el impacto de diferentes tratamientos a dos grupos de pacientes: con angina estable y con infarto de miocardio agudo. Véase por ejemplo Davies et al. (2004). Los resultados de este modelo pueden obtenerse a través del siguiente vínculo del APHO (Association of Public Health Observatoires): http://www.apho.org.uk/apho/models.aspx

\subsection{Suecia}

Suecia cuenta con el modelo SVERIGE, creado para simular el comportamiento en términos demográfico y económico de la población sueca. Se trata de microsimulación dinámico inspirado en el modelo CORSIM. Cuenta con toda la población sueca de 1990 como punto de partida, a partir de la cual se simulan diferentes eventos a lo largo de la vida de los nueve millones de individuos considerados. Entre estos eventos se simula educación, consumo de energía, migraciones, pero también se considera que los individuos enfermen, se jubilen, reciban pensiones, mueran, por lo que se pueden analizar multitud de acciones relacionadas con la Economía de la Salud. Para una revisión de los inicios de la microsimulación sueca, véase Orcutt y Glazer (1980).

\subsection{Uso general}

Por no poder clasificar como de uso específico a un país determinado, incluimos en este epígrafe un modelo desarrollado por la OMS destinado a determinar la carga de diferentes enfermedades a nivel regional y nacional para los países miembros de la OMS. El modelo GBD (Global Burden of Disease) se desarrolló por Murray y López (1997. a, 1997b, 1997c). El modelo utiliza proporciones de riesgo atribuibles a la población, y es capaz de calcular la carga atribuible en una enfermedad (entre las que se incluye CHD) para unos factores de riesgo, población y momento determinados. Para ello se calcula la diferencia entre la carga observada y la que se obtendría si la exposición al riesgo fuera la mínima. El modelo cuenta con cinco componentes: causas de muerte, epidemiología descriptiva de secuelas incapacitantes, carga atribuible a los factores de riesgo seleccionados, proyecciones de carga futura y análisis de sensibilidad. Los factores de riesgo principales considerados son: malnutrición, mala calidad del agua, relaciones sexuales sin protección, ocupación, consumo de tabaco, hipertensión, inactividad física, uso de drogas ilegales y contaminación del aire.

Otro modelo que se puede utilizar a través de la OPS (Organización Panamericana de Salud) es el referente a la gestión de costes de la regionalización de bancos de sangre. Este modelo es muy sencillo, basado en una hoja de cálculo, y las explicaciones de su funcionamiento se pueden obtener en la dirección: www.paho.org/Spanish/AD/ THS/EV/blood guiaCostos.pdf 


\section{Microsimulación «a la carta»}

Los modelos de microsimulación descritos en el epígrafe anterior son el resultado de trabajo que requiere esfuerzos de muchos investigadores y de mucho tiempo de perfeccionamiento. En ocasiones, el investigador no desea emprender un proyecto de gran envergadura para realizar una investigación particular, por lo que también se encuentran en la literatura trabajos en los que la microsimulación constituye una herramienta pero no el objetivo fundamental de la investigación. Es decir, en la construcción de modelos de microsimulación se pretende crear herramientas asequibles para el desarrollo del trabajo de múltiples investigadores. Los esfuerzos se dirigen a partir de una primera versión, a facilitar el funcionamiento, incrementar la rapidez de cálculo, integrar la herramienta de cálculo con otras similares en otros países o dentro del mismo país, ampliar las funciones de la misma. Una vez elaborados estos modelos es fácil obtener resultados en términos de microsimulación sin la pérdida de esfuerzo que implica la creación de la herramienta. Prueba de ello son algunas series de papeles que nacen vinculadas a un esfuerzo microsimulador de gran envergadura, como por ejemplo EUROMOD, que cuenta con una serie de papeles de trabajo (se pueden encontrar en la dirección http://www.econ.cam.ac.uk/dae/mu/emod3.htm), o SAGE dentro de la London School of Economics (http://www.lse.ac.uk/collections/SAGE/discussionPapers.htm).

En otras ocasiones, ese esfuerzo que requiere la construcción de un modelo no compensa al investigador que quiere hacer uso de microdatos pero no obtener una herramienta como output. Rastrear trabajos de estas características es una tarea ardua, ya que los programas individuales utilizados para la microsimulación a la carta y en particular de un investigador no pueden darse a conocer de la misma forma que los modelos descritos en la sección previa. No obstante, desde hace varios años es posible encontrar investigadores utilizando la microsimulación como forma de obtención de resultados que permitan avanzar en el conocimiento de distintas disciplinas, y en particular la salud ya sea desde el punto de vista médico o económico.

La microsimulación se ha aplicado al estudio de cuestiones tan diferentes dentro de la Economía de la Salud como diferentes son las cuestiones que suscitan interés dentro de esta disciplina. Un tema recurrente en esta área de investigación es el análisis de la eficiencia y equidad con las que se realizan los gastos sanitarios. Estas cuestiones, de enorme interés en el general en Economía Pública, lo son mucho más en concreto en el área de la Salud, donde los problemas de eficiencia ligados a la selección adversa y el riesgo moral pueden ser de particular importancia en términos de gasto. De enorme relevancia dentro del estudio de la eficiencia son los análisis coste efectividad, coste eficacia, coste beneficio de las diferentes formas de asignar el gasto sanitario. Asimismo, cuando se habla de equidad, no se debe parar en el análisis de lo que a rentas monetarias se refiere, ya que si a la condición de desfavorecido se une el hecho de que el acceso a los servicios sanitarios es difícil, el problema es mucho más grave. La cuestión del exceso de demanda sanitaria, el coste vinculado al envejecimiento de la población y el tratamiento fiscal favorable de los seguros es también recurrente en el área de Economía de la Salud. 
A continuación, y sin ánimo de ser exhaustivos, se muestran algunos ejemplos de trabajos relativos a estas cuestiones de relevancia en los que se diseñan modelos de microsimulación a la carta, es decir, modelos para la investigación de una cuestión particular, bien porque los investigadores no se encuentran integrados en un equipo de trabajo, porque dichos modelos no existen o porque el desarrollo de un modelo más amplio no tuviera sentido para resolver el problema objeto del estudio.

Los seguros sanitarios han sido objeto de estudio de manera recurrente en la literatura. Chernick el al. (1987) estudian hasta qué punto está vinculado el hecho de que se subsidie el seguro sanitario proporcionado por el empleador y que se contraten seguros por encima del nivel óptimo, creándose en consecuencia un exceso de demanda de servicios sanitarios y un crecimiento rápido del gasto en el sector médico. Para ello, calculan la elasticidad de demanda de los seguros médicos y los servicios médicos mediante un modelo de microsimulación estática aplicado a Estados Unidos. Concluyen que la eliminación de los subsidios provocaría una reducción en la demanda de seguros sanitarios entorno al $20 \%$ y de la demanda de servicios sanitarios entorno al $5 \%$.

También la cuestión de los seguros médicos en USA es abordada en Zabinski el al. (1999), que mediante un modelo de microsimulación que contempla selección adversa, consideran qué ocurriría si se extendiesen las ventajas fiscales de ciertos seguros médicos a todo el mercado. Entre otras conclusiones, obtuvieron que los grupos más perjudicados eran aquellos más pobres y las familias con más hijos. Más recientemente, Pauly y Herring (2000) tratan la cuestión del diseño de una política de primas por parte del empleado que reduzca la ineficiencia ligada al problema de selección adversa. Para ello proponen la «contribución mediante prima eficiente» que ofrecería una cobertura limitada pero más generosa, y lograría la asignación eficiente entre individuos que difieren por razón de riesgo. Aplicado al caso español, López Nicolás (2001) analiza las consecuencias de la política de gastos fiscales ligada a los seguros médicos en España. En López y Vera (2002) se construye una rutina de microsimulación basada en un modelo de elección discreta aplicada a datos de Cataluña para determinar si los subsidios fiscales a los seguros médicos privados se autofinancian. La conclusión es que aplicada al año 1999, la eliminación de dichos subsidios supone un aumento en la recaudación de 69,2 millones de euros anuales, frente a un incremento de coste para el sector público de 8,9 millones de euros anuales.

El análisis de microdatos permite el estudio de una cuestión central en economía como es la desigualdad. Wagstaff y van Doorslaer (1997), pioneros en el estudio de las desigualdades ligadas a la salud, utilizan el método de Aronson para descomponer el efecto redistributivo del sistema de financiación de la salud holandés en los tres componentes: progresividad, equidad horizontal y reordenación.

La investigación médica orientada a la conveniencia o no de determinadas políticas de diagnóstico y tratamiento puede contar con la microsimulación como medio para la toma de decisiones. Como ejemplo, puede citarse el estudio llevado a cabo por Boer el al. (1998) en el que se comparan en términos de coste-efectividad dos posibles modificaciones al programa de diagnóstico de cáncer de mama en Reino Unido: 
acortar el período de revisión de tres a dos años o ampliar la edad de revisión desde los 64 hasta los 69 años. Para ello se simula con microdatos las historias de las mujeres en ausencia de revisión y con cualquiera de las políticas de revisión consideradas. Ambas políticas, con pequeñas diferencias de coste contribuirían a reducciones en la mortalidad.

En van Oortmarssen (1990) se presenta un modelo de diagnóstico de cáncer de mama para Holanda. Cuando se introducen las políticas de diagnóstico adecuadas, el modelo reproduce las tasas de detección y la incidencia del cáncer tal y como se observa en las ciudades holandesas de Utrecht y Nijmegen. El modelo también es capaz de predecir la reducción en la tasa de mortalidad con resultados coherentes con el modelo sueco Kopparberg/Ostergotland. Parámetros clave del modelo son la duración de los estadios preclínicos, y la sensibilidad de la mamografía.

Otro ejemplo de aplicación de técnicas de microsimulación a la investigación médica lo hallamos en Cronin el al. (1998). En este trabajo, y ante la imposibilidad de resolución matemática de problemas complejos, se muestra una aplicación a la detección del cáncer de un modelo de simulación previamente aplicado en el ámbito ingenieril. Para ello se especifica una estructura del modelo y un conjunto de valores de los parámetros, que llevarán aparejados incertidumbre. Se adopta un enfoque Bayesiano y se asume una distribución de probabilidad paramétrica para expresar matemáticamente la incertidumbre ligada a los parámetros. A Partir de aquí, los pasos a seguir son tres. Primero se diseña un experimento de simulación para lograr una buena cobertura del espacio de parámetros. En segundo lugar se modeliza un conjunto de respuesta para el resultado de interés como función de los parámetros del modelo a partir de los resultados de la simulación. En tercer lugar, se resume la variabilidad del resultado de interés, incluyendo la variabilidad debida a la incertidumbre de los parámetros, mediante la combinación de las respuestas posibles y las distribuciones de probabilidad de los parámetros. Este modelo se aplicó a la investigación del efecto del antígeno específico de próstata sobre la tasa de mortalidad de cáncer de próstata.

De aplicación también específica al ámbito hospitalario, encontramos los modelos de simulación desarrollados por Stefen Grover y su equipo (Grover et al. (2000)). Grover es director de análisis coste-efectividad y división de epidemiología en el Hospital General de Montreal, y adscrito al departamento de medicina, epidemiología y bioestadística de la Universidad McGill. Su investigación se centra en el desarrollo y validación de modelos de simulación que predicen la progresión del cáncer y enfermedades cardiovasculares. Los modelos se utilizan para determinar el riesgo y los beneficios de diferentes estrategias de diagnóstico de factores de riesgo e intervenciones terapéuticas.

Más recientemente, en Lubitz el al. (2003), se estima la relación entre el estado de salud de las personas mayores — en particular a los 70 años - y la esperanza de vida y el gasto en salud acumulado desde ese momento hasta la muerte. Para ello se usan datos americanos de 1992 a 1998. La microsimulación es aquí necesaria para estimar la esperanza de vida en distintos estados de salud. El estudio concluyó que los gastos acumulados en salud para mayores con mejor estado de salud a pesar de su ma- 
yor longevidad eran similares a los de peor salud. Las campañas de salud dirigidas a personas de menos de 65 años, pueden mejorar la salud y longevidad sin incrementar los gastos sanitarios.

Para conocer los modelos dedicados a la prevención y tratamiento de enfermedades coronarias (coronary heart disease (CHD)) se puede acudir a Unal et al (2006), donde se lleva a cabo un rastreo exhaustivo por más de 70 publicaciones diferentes. De todos los modelos revisados, mostramos aquí los principales y los que no hayan sido mencionados con anterioridad. Todos ellos comenzaron en todo caso como actuaciones independientes y aunque se hayan desarrollado posteriormente en el seno de instituciones los incluimos aquí por no haber podido acceder ni a su uso ni a sus resultados

1. Modelo CHPD (Coronary Heart Disease Policy). Se trata de un modelo desarrollado en los años 80 . Se utilizó en un principio para analizar las tendencias de mortalidad en CHD y las ganancias en esperanza de vida a partir de modificaciones en los factores de riesgo. También se utilizó para evaluar el coste-efectividad de intervenciones médicas específicas. Basado en los datos de Estados Unidos de América en 1980 relativos a población y mortalidad, consiste en tres submodelos. Un modelo demográfico y epidemiológico que presenta a la población sana entre 35 y 84 años categorizada por sexo, edad y riesgo de enfermedad cardiovascular. Otro modelo puente que cubre a los individuos que enferman durante los primeros 30 días de enfermedad, y un último modelo de historia de la enfermedad, que incluye a aquellos que sobreviven 30 días siguiéndolos a lo largo del tratamiento. El modelo permite al usuario simular los efectos sobre la enfermedad de una intervención bien de tipo modificación en los factores de riesgo (tabaco, colesterol, peso) o terapéuticas e inferir los efectos sobre mortalidad, morbilidad y costes hasta 30 años después.

2. El modelo PREVENT se desarrolló en Holanda por Gunning-Schepers durante los años ochenta. Se ha utilizado para estimar los beneficios en salud de cambios en la prevalencia de factores de riesgo (tabaco, colesterol, hipertensión, obesidad, actividad física y consumo de alcohol) mediante la comparación de la continuación de las tendencias existentes con una alteración de las proporciones de población con factores de riesgo dados. El modelo permite asociar un factor de riesgo con más de una enfermedad y viceversa. La evolución demográfica también se tiene en cuenta en las simulaciones.

3. El modelo de esperanza de vida cardiovascular (Cardiovascular Life Expectancy Model) se desarrolló primeramente en Canadá por Grover et al en 1992 con el fin de analizar el coste-efectividad de distintas opciones de tratamiento de CHD. Desde 1998 en adelante se describe como un modelo de Markov. El modelo incluye una primera etapa CHD en la que se calcula la probabilidad de muerte (por CHD u otras causas) y el riesgo anual de un evento relacionado con CHD (con y sin intervención) para una persona asintomática de 
CHD al entrar en el modelo. Los factores de riesgo considerados son edad, sexo, presión sanguínea diastólica, colesterol, hipertrofia ventricular izquierda, intolerancia a la glucosa y consumo de tabaco. Tras desarrollar CHD, una persona pasa a la segunda etapa del modelo en la que se calcula el riesgo de muerte durante doce meses tras un infarto de miocardio. La diferencia entre la mortalidad acumulada predicha con y sin intervención durante el resto de vida esperada representa los años de vida ganados tras la intervención.

4. El modelo IMPACT CHD de mortalidad se desarrolla en primer lugar por Capewell y sus colegas en 1996. (Capewell et al (1999)). Utilizando un ahoja de cálculo MS EXCEL, combina datos de muchos recursos referentes a número de pacientes, tratamientos y su efectividad, tendencia en factores de riesgo, y efectos sobre la mortalidad. Las muertes impedidas y/o retrasadas se pueden estimar, por lo que se puede estimar la variación en la tasa de mortalidad a lo largo de un período de tiempo achacable a cambios e los tratamientos y en los factores de riesgo (consumo de tabaco, colesterol, presión sanguínea, obesidad, actividad física, diabetes). Otros output que se pueden extraer del modelo son los años de vida ganados y el coste-efectividad de intervenciones específicas.

\section{Reflexiones finales}

La revisión que se ha presentado en las páginas anteriores pone de manifiesto que la microsimulación está cobrando peso en multitud de ámbitos de la Economía de la Salud, ya sea de forma coordinada o mediante el trabajo individualizado. Las críticas achacables a los modelos creados a la carta son resultado de su naturaleza. Por el hecho de estar creados para la resolución de un problema específico no es posible comprobar qué ocurre si los factores que afectan o determinan el problema a analizar son diferentes a los contemplados en el modelo. Además, al crearse de forma independiente y no en el ámbito de instituciones públicas, no se ponen al servicio del público en general, bien sea porque no se difunde su existencia, no se saben manejar, o simplemente se trata de un trabajo no compartido. Debido a que se construyen de forma particular, no se difunde suficientemente la información que permitiría replicar su construcción, lo que impide a menudo validar los resultados y hace que funcionen como cajas negras.

Esta reflexión debería servir para que los recursos públicos se pusieran a disposición de aquellos que trabajan en el ámbito de la microsimulación en salud y favorecer así aquello que el trabajo individualizado no logra: el acceso a datos de calidad, el aunar los esfuerzos de expertos que permitan la elaboración de herramientas sólidas, la transparencia de los métodos, básica para la confianza en los resultados y el poner a disposición de los investigadores las herramientas elaboradas. 


\section{Referencias bibliográficas}

[1] BOER R., DE KONING H., THRELFALL A., WARMERDAM P., STREET A., FRIEDMAN E., WOODMAN C. (1998) «Cost Effectiveness of Shortening Screening Interval or Extending Age Range of NHS Breast Screening Programme: Computer Simulation Study». British Medical Journal, 317 (7155): 376-379.

[2] BROWN, L. and A. HARDING (2002). «Social Modelling and Public Policy: Application of Microsimulation Modelling in Australia». Journal of Artificial Societies and Social Simulation, 5, (4) . http://jasss.soc.surrey.ac.uk/5/4/6.html

[3] BROWN, L., ABELLO, A., PHILLIPS, B. AND A. HARDING, (2004) «Moving towards an Improved Microsimulation Model of the Australian Pharmaceutical Benefits Scheme». The Australian Economic Review 37 (1): 41-61.

[4] CAPEWELL S, MORRISON CE, MCMURRAY JJ. (1999) «Contribution of modern cardiovascular treatment and risk factor changes to the decline in coronary heart disease mortality in Scotland between 1975 and 1994». Heart.81:380-386.

[5] CHERNICK H.A., HOLMER M.R. and D.H. WEINBERG DH. (1987) «Tax policy toward health insurance and the demand for medical services». Journal of Health Economics; 6(1):1-25.

[6] CRONIN K, LEGLER JM, ETZIONI R. (1998) «Assessing uncertainty in microsimulation modeling with application to cancer screening interventions». Statistics in Medicine 17:2509-2523.

[7] CURRY, C. (1996) «PENSIM: A Dynamic Simulation Model of Pensioners' Incomes», Government Economic Service Working Paper no.129

[8] DAVIES R, NORMAND C, RAFTERY J, RODERICK P, SANDERSON C. (2004) «Policy analysis for coronary heart disease: a simulation model of interventions, costs and outcomes» Report to the Department of Health, July 2003 (revised April 2004). London: London School of Hygiene and Tropical Medicine, 2004.

[9] EVANDROU, M., FALKINGHAM, J., JOHNSON, P. and K. RAKE (2001). «SAGE: Simulating Social Policy for an Ageing Society. A Research Agenda.SAGE Discussion Paper n. ${ }^{\circ}$ 1. http://www.lse.ac.uk/collections/SAGE/pdf/SAGE DP1.pdf

[10] FAVREAULT, M. and SMITH, K. (2004) A Primer on the Dynamic Simulation of Income Model (DYNASIM3). Washington, DC: The Urban Institute. http://www.urban.org/UploadedPDF/410961 Dynasim3Primer.pdf

[11] FREDRIKSEN D. (1998). Projections of Population, Education, Labour Supply and Public Pension Benefits: Analyses with the Dynamic Microsimulation Model MOSART. Social and Economics Studies, Statistics Norway, Oslo.

[12] GROVER SA, COUPAL L, ZOWALL H, RAJAN R, TRACHTENBERG J, ELHILALI M, et al. (2000) «The economic burden of prostate cancer in Canada: forecasts from the Montreal Prostate Cancer Model». Canadian Medical Association Journal 162(7):987-92

[13] GRUBER, J. (2000). «Microsimulation Estimates of the Effects of Tax Subsidies for Health Insurance», National Tax Journal, 53(3), Part I, 329-342. http://ntj.tax.org/wwtax/ntjrec.nsf/233E3132EB19A9E485256AFC007F257B/SFILE/v 53n3p1329.pdf

[14] HANCOCK, R., MALLENDER, J. and PUDNEY, S. (1992) «Constructing a ComputerModel for Simulating the Future Distribution of Pensioners' Incomes forGre- 
at Britain» in Hancock, R. and Sutherland, H. (editors) MicrosimulationModels for Public Policy Analysis: New Frontiers, London: STICER. Pages 33-66.

[15] KING, A. BÆKGAARD, H. and M. ROBINSON (1999) «DYNAMOD-2: An overview». Technical Paper no. 19. NATSEM University of Canberra. http://www.natsem.canberra.edu.au/pubs/tps/tp19/tp19.pdf

[16] LÓPEZ NICOLÁS, A. (2001). «Seguros sanitarios y gasto público en España. Un modelo de microsimulación para las políticas de gastos fiscales en sanidad» Papeles de Trabajo del Instituto de Estudios Fiscales (Serie Economía) n. ${ }^{\circ} 12 / 2001$. Instituto de Estudios Fiscales. Madrid.

[17] LÓPEZ NICOLÁS, A. y M. VERA (2002) «Are Tax Subsidies for Private Medical Insurance Self-financing? Evidence from a Microsimulation model for Outpatient Episodes in a NHS context» Working Paper 632. Dept. Economics and Business. Universitat Pompeu Fabra.

[18] LUBITZ, J., LIMING C., KRAMAROW, E. and H. LENTZNER (2003) «Health, Life Expectancy, and Health Care Spending among the Elderly» The New England Journal of Medicine 349:1048-1055 September 11, 2003.

[19] MORRISON R. and B. DUSSAULT (2000). «A full-fledged Canadian actuarial stochastic model designed for the fiscal and policy analysis of social security schemes». http://www.actuaries.org/members/en/committees/SOCSEC/documents/dynacan.pdf

[20] MURRAY C.J. and A.D. LÓPEZ. (1997a) «Alternative projections of mortality and disability by cause 1990-2020: Global Burden of Disease Study». Lancet. 349:14981504.

[21] MURRAY C.J. and A.D. LÓPEZ. (1997b) «Global mortality, disability, and the contribution of risk factors: Global Burden of Disease Study». Lancet. 349:1436-1442.

[22] MURRAY C.J. and A.D. LÓPEZ. (1997c) «Mortality by cause for eight regions of the world: Global Burden of Disease Study». Lancet.. 349:1269-1276.

[23] NELISSEN, J. (1993). «Labour market, income formation and social security in the microsimulation model NEDYMAS». Economic Modelling, 10 (3): 225-272.

[24] NELISSEN, J. (1996) «Social Security and Lifetime Income Redistribution: a microsimulation approach» in Harding, A (ed) Microsimulation and Public Policy. (Amsterdam: North-Holland).

[25] VAN OORTMARSSEN GJ, HABBEMA JD, VAN DER MAAS PJ, DE KONING HJ, COLLETTE HJ, VERBEEK AL, et al. (1990) «A model for breast cancer screening». Cancer 1990; 66: 1601-1612

[26] ORCUTT. H. and A. GLAZER (1980). «Microanalytic Modelling and Simulation». In B. Bergmann, G. Eliasson and G. H. Orcutt, editors, Micro Simulation Models: Methods and Applications. IUI Conference Reports 1980:1, Industrial Institute for Economic and Social Research, Stockholm.

[27] PAULY M.V., and B.J. HERRING (2000) «An efficient employer strategy for dealing with adverse selection in multiple-plan offerings: an MSA example» Journal of Health Economics 19 (4): 513-528.

[28] SPIELAUER (2002a) «Dynamic Microsimulation of Health Care Demand, Health Care Finance and the Economic Impact of Health Behavior. Part I: Background and a Comparison with Cell-Based Models». - IIASA IR-02-032

[29] SPIELAUER (2002b) «Dynamic Microsimulation of Health Care Demand, Health Care Finance and the Economic Impact of Health Behavior. Part II: Survey and Re- 
view». IIASA IR, 2002. http://www.iiasa.ac.at/Publications/Documents/IR-02036.pdf

[30] SUTHERLAND, H., ed. (2001), «EUROMOD: An Integrated European BenefitTax Model» (Final Report) EUROMOD Working Paper No. EM9/01, June.

[31] UNAL, B., S. CAPEWELL and J.A. CRITCHLEY (2006) «Coronary heart disease policy models: a systematic review» BMC Public Health. 2006; 6: 213.

[32] WAGSTAFF, A. and E. VAN DOORSLAER (1997) «Progressivity, horizontal equity and reranking in health care finance: a decomposition analysis for the Netherlands» Journal of Health Economics, 16 (5): 499-516.

[33] ZABINSKI, D., SELDEN T.M., MOELLER, J.F. and J.S. BANTHIN (1999) «Medical savings accounts: Microsimulation results from a model with adverse selection». Journal of Health Economics 18 (2): 195-218.

[32] ZAIDI, A. and K. RAKE (2002) «Dynamic Microsimulation Models: A Review and some Lessons from SAGE» SAGE Discussion paper no.2. ESRC-Sage Research Group, LSE SAGE/DP2 http://www.lse.ac.uk/collections/SAGE/pdf/SAGE DP2.pdf 